\title{
Polarization-diversity silicon photonic Mach-Zehnder delay interferometer for optical signal processing integrated with a phase-controllable polariza- tion beam splitter
}

\author{
Takahiro Arai ${ }^{1}$ and Hiroyuki Uenohara ${ }^{1, \text { a) }}$
}

\begin{abstract}
This study investigates a polarization-diversity Mach-Zehnder delay interferometer (MZDI) for optical signal processing integrated with a polarization beam splitter (PBS) using silicon photonics technology. The PBS consists of two-stage MZDIs. By using the birefringence of the silicon waveguide and controlling the phase with thermal heaters on MZDIs, the polarization extinction ratio (PER) of the PBS was improved. MZDIs with a free spectral range (FSR) of $40 \mathrm{GHz}$ in both TE and TM modes were designed using the group index for both polarization modes. The PER of 40-GHz-FSR MZDIs for TE and TM modes of 15 and $10 \mathrm{~dB}$, respectively, within the C-band were observed.

Keywords: polarization diversity, silicon photonics, Mach-Zehnder delay interferometer

Classification: Integrated optoelectronics (lasers and optoelectronic devices, silicon photonics, planar lightwave circuits, polymer optical circuits, etc.)
\end{abstract}

\section{Introduction}

Silicon photonics has received significant attention owing to the small size, high-density integration, and low power consumption capability [1]. In recent times, heterogeneous integration for III-V active components has been achieved on a Si platform [2, 3], large-scale $32 \times 32$ and $50 \times 50$-nonblocking switches have been reported $[4,5]$, and a coherent receiver consisting of optical splitters and $\mathrm{Ge}$-photodetectors has been integrated into a single chip [6,7]. Additionally, we previously investigated an optical serial-to-parallel converter (OSPC) consisting of Mach-Zehnder delay interferometers (MZDIs, hereinafter referred to as MZDI-OSPC) [8, 9]. The operating principle is shown in Fig. 1. We used a one-bit preamble with an optical phase shift of $\pi / 2$ at the beginning of the optical packets, and a MZDI with a delay line length corresponding to the propagation distance of light in the $N$ bit duration with a phase bias of $-\pi / 2$. By receiving output from MZDIs with balanced photodetectors, only the $N$-th bit could be extracted from one period of the optical packet, and other symbols were cancelled in the case of binary code (onoff keying (OOK) and binary/differential phase-shift keying (BPSK/DPSK)) [10, 11]. However, owing to the high bire-

${ }^{1}$ Laboratory for Future Interdisciplinary Research of Science and Technology, Tokyo Institute of Technology, 4259 Nagatsuta, Midori-ku, Yokohama, Kanagawa 226-8503, Japan

a) uenohara.h.aa@m.titech.ac.jp

DOI: 10.1587/elex.17.20200176

Received May 14, 2020

Accepted May 22, 2020

Publicized June 3, 2020

Copyedited June 25, 2020

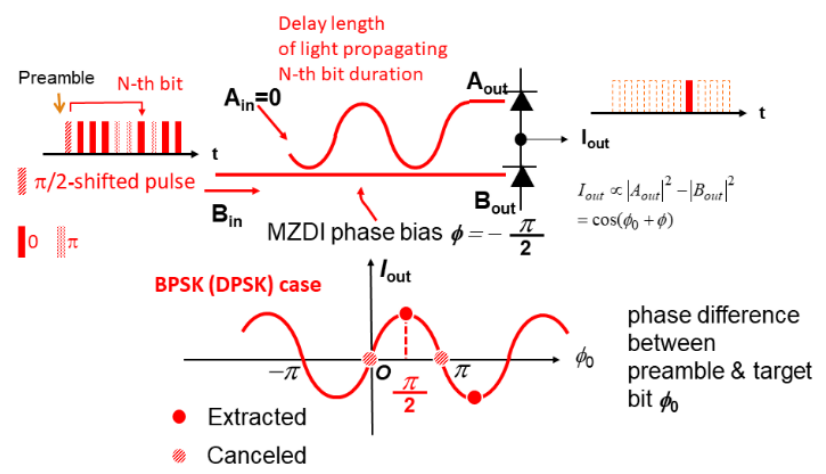

Fig. 1 Operating principle of a MZDI-type optical serial-to-parallel converter for $\mathrm{N}$-th bit extraction.

fringence of a silicon waveguide, polarization-independent operation becomes a technical issue, particularly in passive devices. To overcome this, the introduction of a rib waveguide is effective [12], but the bending radius should be larger than that of the silicon waveguide. The polarization diversity scheme is another candidate, and a polarization splitter (PS) and rotator (PSR) integrated with optical switches and an optical modulator for both polarizations have been reported $[4,6,7,13]$. The directional-coupler-based PS utilizes the difference of coupling length due to birefringence, and integrated PSR is based on the rotated inherent polarization axes due to the asymmetric shape, and a small size of approximately $10 \mu \mathrm{m}$ for the former [14] and 3-5 $\mu \mathrm{m}$ for the latter can be achieved [15]. However, PER is not as large as $15 \mathrm{~dB}$ within the whole $\mathrm{C}$-band. In addition, a fine process is required to maintain a large PER within a wide bandwidth, and thus, a fabrication tolerant structure is desirable.

We have previously investigated a MZDI-type polarization beam splitter (PBS, hereafter referred to as MZDI-PBS) for use in polarization diversity operation [16]. There are several types of PBSs, such as the asymmetric waveguide directional coupler (DC) $[17,18,19,20]$ bending coupler-type DC [21, 22, 23, 24], plasmonic waveguide DC [23], multimode interferometer (MMI) [25, 26], cascaded MMI [27], and MMI with subwavelength gratings [28]. A wide range of wavelengths over the C-band and a large polarization extinction ratio (PER) of $>20 \mathrm{~dB}$ have been reported using the bending DC structure, and a very small size has been achieved using a bending DC with plasmonic waveguides. However, these devices require fine designs, and tolerable structures would be desirable. Compared to these structures, 
PER can be controlled in an MZDI-type structure, because a thermo-optic (TO) heater can be installed on the device and phase conditions can be tuned to obtain the maximum PER [29, 30, 31, 32, 33].

In this study, MZDI-OSPCs integrated with a two-stage MZDI-PBS are investigated. Orthogonal polarized lights (TE and TM components) are split over the C-band, and transmissivity spectra for TE and TM are obtained with orthogonal polarization suppression. The preliminary results are presented herein, and the cause of the performance degradation is also discussed.

\section{Device structures and operating principles}

The schematic of the polarization-diversity silicon photonic MZDI-OSPCs integrated with a two-stage MZDI-PBS is shown in Fig. 2. Owing to the birefringence of a silicon waveguide, the PBS is based on an MZDI with a total delay length $\Delta \mathrm{L}$, corresponding to the out-of-phase and in-phase conditions for the TE and TM modes, respectively. $\Delta \mathrm{L}$ is expressed by Eq. (1):

$$
\Delta L=\frac{N \cdot \lambda}{2\left(n_{g-T E}-n_{g-T M}\right)},
$$

where $N$ is an odd number $(N=1,3,5, \ldots), \lambda$ is the carrier wavelength of a signal, and $n_{g-T E}$ and $n_{g-T M}$ are the group indices of a silicon waveguide for the TE and TM modes, respectively. To design a PBS free spectral range (FSR) that is greater than the bandwidth of the C-band (30 $\mathrm{nm}$ ), the value of $N$ was set to 3 , and thus, $\Delta L$ was $3.15 \mu \mathrm{m}$. The input light with TE and TM modes is injected into the upper port of the MZDI-PBS, and the TE and TM modes are supposed to be output from the upper and lower output ports. To enhance the PER of the PBS, the second-stage MZDIPBSs are connected to the two output ports of the first-stage MZDI-PBS of the same size. The upper output port of the upper second-stage MZDI-PBS and the lower output port of the lower second-stage MZDI-PBS were used to monitor the PBS output power. The lower output port of the upper second-stage MZDI-PBS is connected to an MZDI-OSPC for the TE mode, and the upper port of the lower secondstage MZDI-PBS is connected to that of the TM mode. The output ports are numbered from one to six from the top to the bottom, as shown in Fig. 2. The FSR of the MZDI-OSPCs was designed to be $40 \mathrm{GHz}$. The delay line lengths of the MZDI-PBSs were calculated to be $1.785 \mathrm{~mm}$ and $2.146 \mathrm{~mm}$ for the TE and TM modes, respectively; this is achieved by using the group indices of 4.2 and 3.5 for both modes. The bending radius for connecting the straight waveguides of the delay lines was $10 \mu \mathrm{m}$. The waveguide was fabricated on a silicon on insulator (SOI) substrate, and a silicon waveguide of $0.45 \mu \mathrm{m}$ width and $0.21 \mu \mathrm{m}$ thickness was used. It was embedded within the upper and lower $\mathrm{SiO}_{2}$ cladding layers of $\sim 2.0 \mu \mathrm{m}$ thickness. All the MZDIs had $2 \times 2$ MMI-type optical splitters and couplers of $1.8 \mu \mathrm{m}$ width on the input and output sides. The MMI length for the MZDI-PBS was $12.5 \mu \mathrm{m}$ for a 50:50 splitting ratio, and that for the MZDIOSPCs was $11.0 \mu \mathrm{m}$ to obtain an asymmetric splitting ratio, equalizing the loss in the shorter and longer waveguides. The TiN heaters were installed above the silicon waveguide in the

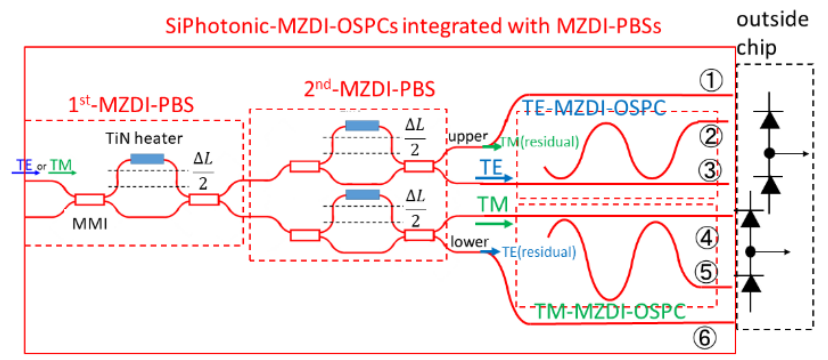

Fig. 2 Schematic of silicon photonic MZDI-OSPCs for TE and TM modes integrated with a two-stage MZDI-PBS.

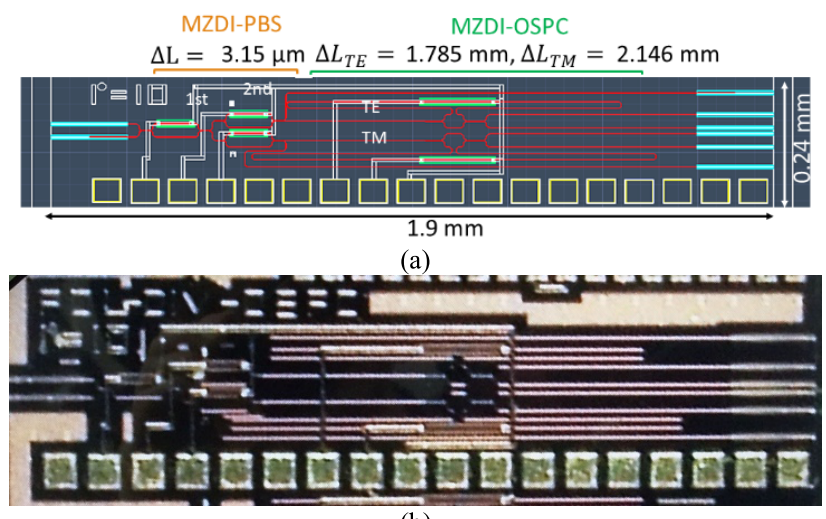

(b)

Fig. 3 Fabricated device. (a) CAD layout; (b) photograph.

MZDI-PBSs of $100 \mu \mathrm{m}$ length, and their series resistances were approximately $200 \Omega$ each. Spot size converters were loaded at the input and output ports, and the coupling loss was about $2 \mathrm{~dB} /$ facet. The propagation loss of the waveguide was typically about $2 \mathrm{~dB} / \mathrm{cm}$. The total device size was 400 $\mu \mathrm{m} \times 1.9 \mathrm{~mm}$. The computer-aided design (CAD) layout and a photograph of the device are shown in Figs. 3(a) and (b).

\section{Experimental results}

The characteristics of the fabricated device were measured by injecting the light from a white light source and monitoring the output power of the device. The transmissivity was evaluated using an optical spectrum analyzer, and the polarization of the injection light was set with a polarizer and a polarization controller.

\subsection{Setting heater voltages of PBS}

Prior to the transmissivity of the MZDI-OSPCs, the heater voltages of the MZDI-PBS were set to maximize the PER of the device. In the case of no control in all the heaters, the injected TE and TM mode lights were obtained as output from the reverse side of the expected positions. The near field pattern (NFP) of the device for the TE and TM modes are indicated in Figs. 4(a) and (b), respectively. The TE mode is expected to be output from output ports 2 , and 3 (and residual TE from port 6), but it was done with comparatively higher power from output ports 4,5 , and 6 . To recover the performance of the device, the heater voltages of the PBS were controlled. Figure 5(a) indicates the TE mode output power of the device for the case in which the heater voltage 


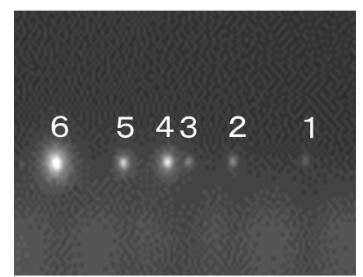

(a)

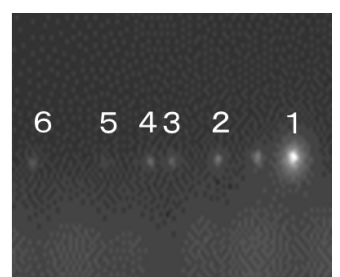

(b)
Fig. 4 NFP of the device without heater control. (a) TE input; (b) TM input.

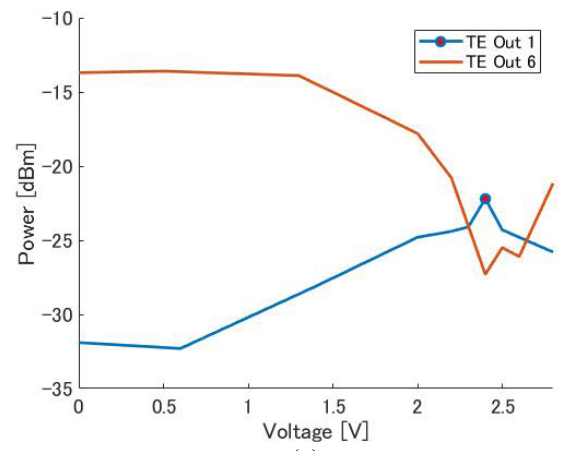

(a)

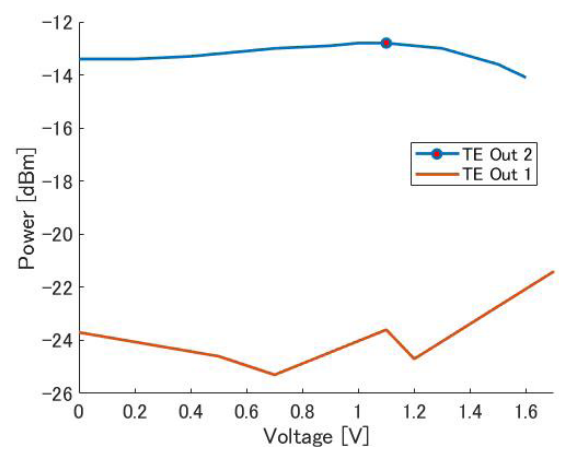

(b)

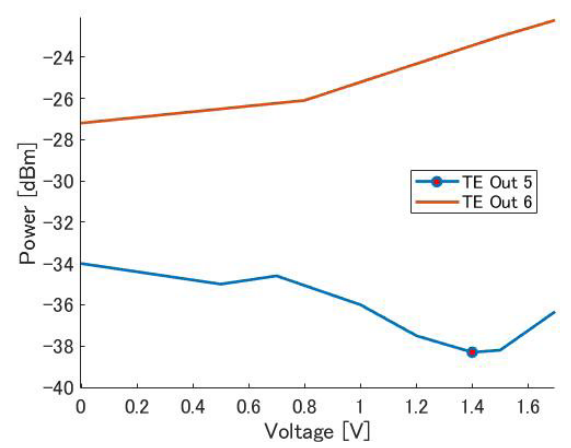

(c)

Fig. 5 Output power of the device in the case of heater voltage control. (a) The heater voltage of the first-stage MZDI-PBS alone was changed. (b) Heater voltage of the upper second-stage MZDI-PBS was changed, and the voltage of the first-stage MZDI-PBS was fixed at $2.4 \mathrm{~V}$. (c) The heater voltage of the lower second-stage MZDI-PBS was changed, and the voltage of the second-stage MZDI-PBS was fixed at $2.4 \mathrm{~V}$. In all cases, the polarization of the input light was TE.

of the first-stage MZDI-PBS was changed while that of the second-stage MZDI-PBS was not controlled (i.e., $0 \mathrm{~V}$ ). At $0 \mathrm{~V}$, the TE mode was output from port 6 with larger level than the residual output, and it was reversed compared to the expected condition. However, at $2.4 \mathrm{~V}$, the TE mode was output with maximum PER from output port 1, although

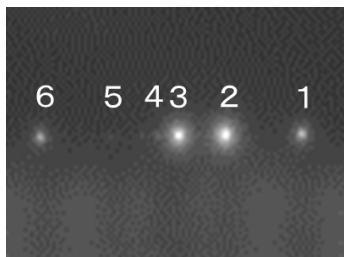

(a)

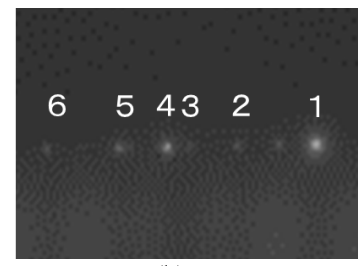

(b)
Fig. 6 NFP of the device under the condition of the optimized heater voltages. (a) TE mode; (b) TM mode.

the PER was not large. To further improve the PER of the following MZDIs, heater voltages of the second-stage MZDI-PBSs were controlled. For the upper second-stage MZDI-PBS, voltage was set to maximize and minimize the output power from the output ports 2 and 1 , then the residual TM output should be maximized from the output port 1 . Similarly, for the lower second-stage MZDI-PBS, voltage was set to minimize and maximize the output power from the output ports 5 and 6 , and then residual TE output should be maximized from the output port 6 . Finally, applied voltage to the upper and lower second-stage MZDI-PBSs were decided to be $1.1 \mathrm{~V}$ and $1.4 \mathrm{~V}$, respectively, as shown in Figs. 5(b) and (c). Contrary to that as shown in Fig. 4, the NFP from the device could be controlled such that the TE and TM modes were obtained as output with higher power from ports 2, 3 and 4, 5, respectively, as indicated in Figs. 6(a) and (b). The residual TM output from port 1 was high enough to increase PER in the upper MZDI-OSPC. To improve the PER, the splitting ratio of the MMIs should be precisely redesigned.

\subsection{Transmissivity of MZDI-OSPCs}

Next, the transmissivity of the MZDI-OSPCs was evaluated. With a wide wavelength range (the entire C-band), the transmissivity for the TE mode from output ports 2 and 3 (upper MZDI-OSPC) was approximately $20 \mathrm{~dB}$ larger than that from output ports 4 and 5 (lower MZDI-OSPC), as shown in Fig. 7(a). In Fig. 7(b), by contrast, the transmissivity for the TM mode from output ports 4 and 5 (lower MZDI-OSPC) was approximately $10 \mathrm{~dB}$ larger than that from output ports 2 and 3 (upper MZDI-OSPC). In addition, the transmissivity of the upper MZDI-OSPC was $20 \mathrm{~dB}$ larger for the TE mode compared with that for the TM mode, and the transmissivity of the lower MZDI-OSPC was $15 \mathrm{~dB}$ larger for the TM mode compared with that for TE mode. In both cases, the maximum transmissivity of the upper and lower MZDI-OSPCs were compared. When operating MZDI-OSPCs for OSPC processing, subtraction of the upper output from the lower one corresponds to the output signal strength. Therefore, the subtracted transmissivity of port 3 from port 2 and port 5 from port 4 were calculated. The results are shown in Fig. 8. The significant feature is the ratio of the maximum transmissivity of port 2 - port 3 to port 4 - port 5 , or the ratio of TE to TM. From these results, the polarization was effectively separated between the upper and lower MZDI-OSPCs for both the TE and TM modes. It is noted that, in Figs. 7, 8 , and 9 , transmissivity from fiber to fiber is evaluated, and the polarization dependence of fiber coupling, propagation, bending, and MMI splitting ratio are included. At the present stage, loss factors for TE and TM modes were designed to 


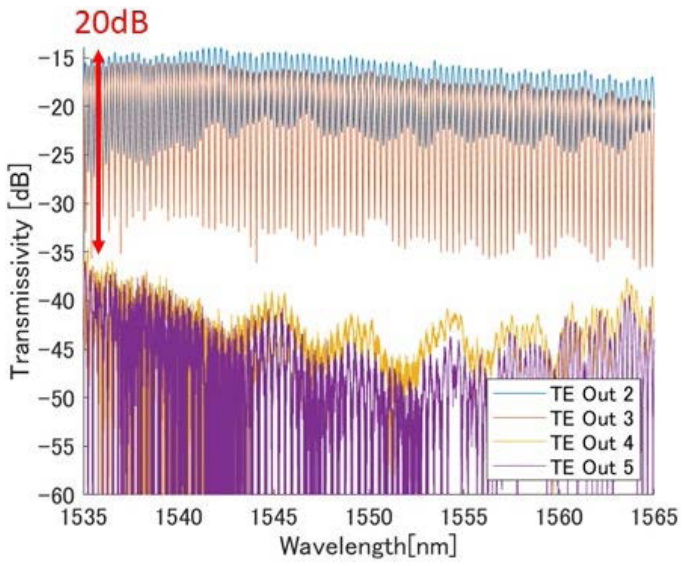

(a)

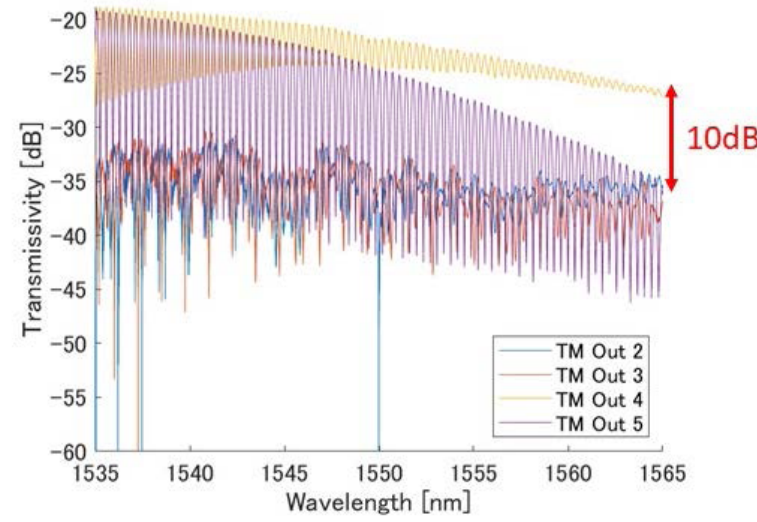

(b)

Fig. 7 Transmissivity spectra of MZDI-OSPCs. (a) Wide wavelength range for TE input; (b) wide wavelength range for TM input.

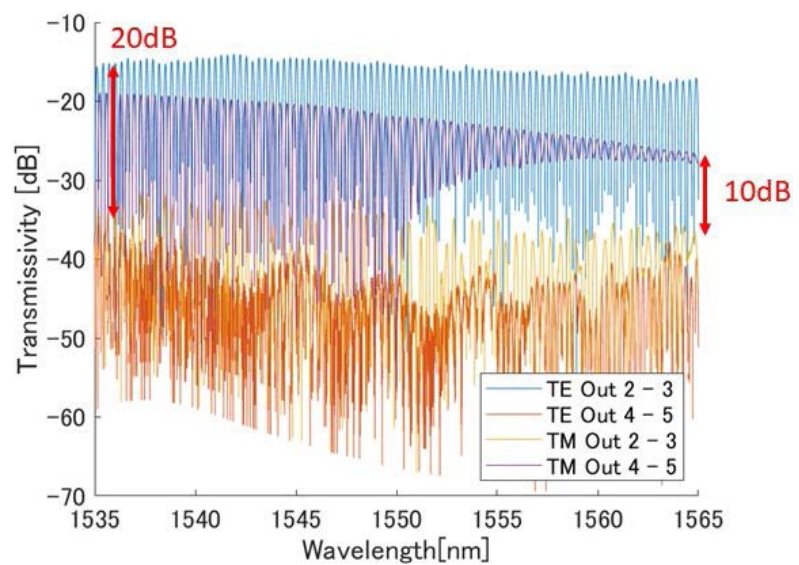

Fig. 8 Transmissivity spectra obtained by calculating the transmissivity from output ports 2-3 and output ports 4-5.

be small, but there is some room for improvement.

For the TM mode, as shown in Fig. 7(b), transmissivity from output port 5 was drastically degraded at longer wavelengths compared to that from output port 4 . The peakto-valley ratio from output port 4 became very small at the same wavelength range. This is considered to be due to the power splitting ratio of the MMI for the TM mode deviating from 50:50, although that for TE mode was almost constant within the C-band. The wavelength and polarization depen-

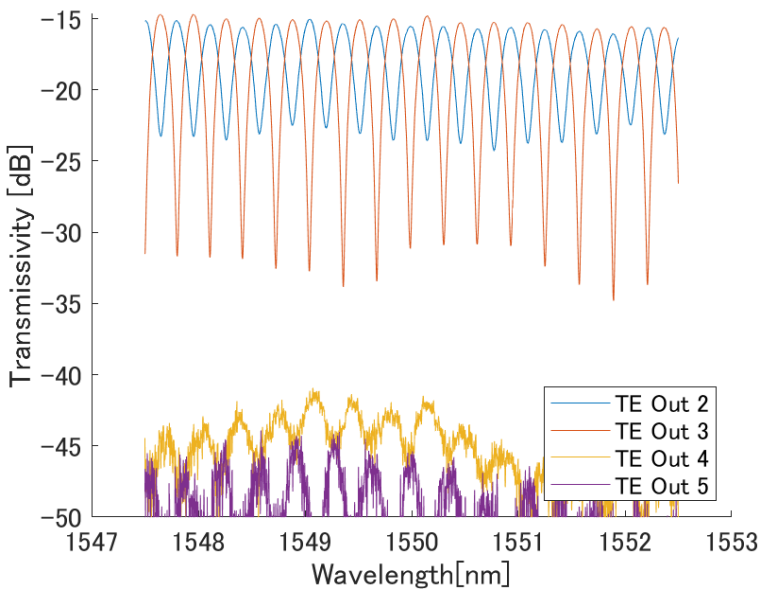

(a)

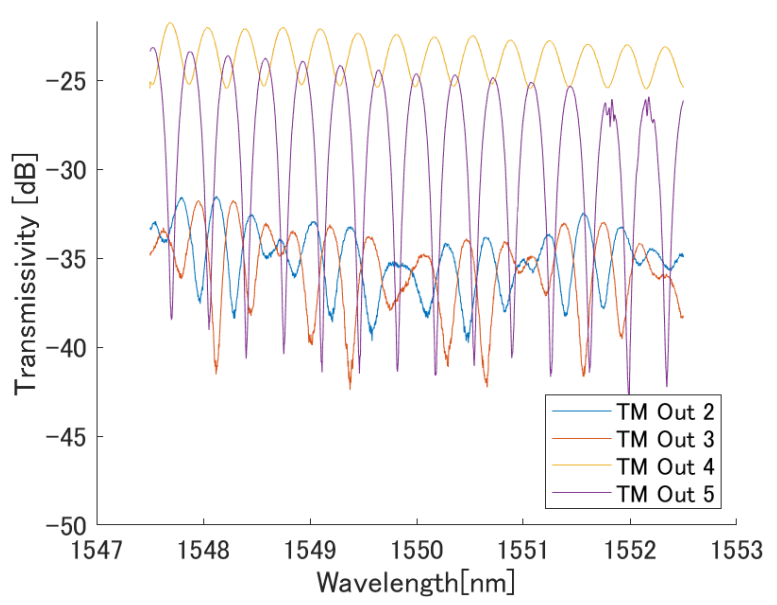

(b)

Fig. 9 Transmissivity spectra of MZDI-OSPCs. (a) Narrow wavelength range for TE input; (b) narrow wavelength range for TM input.

Table I FSR and group index of the device.

\begin{tabular}{|l|l|l|l|l|l|}
\hline Spectral & Simulation & Out 2 & Out 3 & Out 4 & Out 5 \\
\hline FSR (nm) & 0.320 & 0.3153 & 0.3150 & 0.3550 & 0.3567 \\
\hline Error rate (\%) & & -1.5 & -1.5 & 10.9 & 11.5 \\
\hline Group refractive index & $\begin{array}{l}4.2(\mathrm{TE}) \\
3.5(\mathrm{TM})\end{array}$ & 4.26 & 4.26 & 3.15 & 3.14 \\
& \multicolumn{1}{|l|}{} & & & \\
\hline
\end{tabular}

dence of the splitting ratio are prominent technical issues in the case of MMI. The transmissivity spectra for both the upper and lower MZDI-OSPCs for the TE and TM modes are indicated in Figs. 9(a) and (b) in a narrow wavelength range. The FSR of the transmissivity spectra was designed to be $40 \mathrm{GHz}$, corresponding to a wavelength spacing of $0.32 \mathrm{~nm}$. From the measured FSRs, the group indices of the silicon waveguide for the TE and TM modes can be evaluated. The summarized results are shown in Table I. The deviation of the FSR and thus the group index for TE modes are $-1.5 \%$, but those for the TM mode are approximately $+11 \%$. The FSR could be improved by redesigning the delay line length. Differential group delay should be compensated, and it would be done by designing the equal optical lengths between TE-/TM-MZDI-OSPC and the balanced photodetector, and by using the electronic signal processing after the 
balanced photodetection.

\section{Conclusions}

In conclusion, silicon photonic MZDI-OSPCs were fabricated for use in optical signal-to-parallel processing and integrated with two-stage MZDI-PBSs, and the device performance was evaluated. TO heaters installed on silicon waveguides in the PBS parts enabled the recovery of the PER of the device. By controlling the heater voltages of the TO heaters for the PBS, the PER could be optimized. Thus, the PER of the following MZDI-OSPC with FSR $=40 \mathrm{GHz}$ (at $15 \mathrm{~dB}$ and $10 \mathrm{~dB}$ for TE and TM modes, respectively) were obtained within the C-band. Because of the deviation of the power splitting ratio of the MMIs from 50:50 for the TM mode, the PER and peak-to-valley ratios were degraded. By redesigning the MMI lengths or introducing wavelengthinsensitive power splitters, the PER of the device could be improved, and a low-polarization-sensitive operation could be achieved.

\section{Acknowledgments}

We would like to acknowledge Prof. Emeritus K. Iga, Prof. K. Kobayashi, Prof. F. Koyama and Assoc. Prof. T. Miyamoto for their encouragement and discussions. This work was supported by the Strategic Information and Communications R\&D Promotion Programme, Ministry of Internal Affairs and Communications \#MIC/SCOPE 162103103.

\section{References}

[1] H. Yamada, et al.: "Si photonic wire waveguide devices," IEEE J. Sel. Top. Quantum Electron. 12 (2016) 1371 (DOI: 10.1109/ jstqe.2006.880611).

[2] M.J.R. Heck, et al.: "Hybrid silicon photonics integrated circuit technology,” IEEE J. Sel. Top. Quantum Electron. 19 (2012) 6100117 (DOI: 10.1109/jstqe.2012.2235413).

[3] Y. Urino, et al:: "First demonstration of athermal silicon optical interposers with quantum dot lasers operating up to $125^{\circ} \mathrm{C}$," J. Lightw. Technol. 33 (2015) 1223 (DOI: 10.1109/j1t.2014.2380811).

[4] K. Suzuki, et al.: "Nonduplicate polarization diversity $32 \times 32$ silicon photonics switch based on a SiN/Si double-layer platform," J. Lightw. Technol. 38 (2020) 226 (DOI: 10.1109/j1t.2019.2934763).

[5] S. Han, et al:: "50 $\times 50$ polarization-insensitive silicon photonic MEMS switches: design and experiment," Proc. 42nd European Conf. Optical Communication (ECOC2016) (2016) Th.3.A.4.

[6] C. Doerr, et al:: "Single-chip silicon photonics 100-Gb/s coherent transceiver," Proc. Optical Fiber Commun. Conf. 2014 (OFC2014) (2014) Th5C.1 (DOI: 10.1364/ofc.2014.th5c.1).

[7] S. Yamanaka, et al:: "Silicon photonics coherent optical subassembly with EO and OE bandwidth s of over 50GHz," Proc. Optical Fiber Commun. Conf. 2020 (OFC2020) (2020) Th4A.4 (DOI: 10.1364/ofc.2020.th4a.4).

[8] H. Kusano, et al.: "Investigation of a silicon photonic optical serialto-parallel converter with Mach-Zehnder delay interferometers and integrated Ge-photodetectors," IEEE J. Quantum Electron. 51 (2015) (DOI: 10.1109/jqe.2015.2443376).

[9] K. Aramaki and H. Uenohara: "Tolerable performance of silicon photonic serial-to-parallel converter with variable power splitter," Opt. Express 26 (2018) 7740 (DOI: 10.1364/oe.26.007740).

[10] G. Yazawa, et al.: "Optical serial-to-parallel conversion technique with phase-shifted preamble for optical label switching systems," IEEE J. Quantum Electron. 47 (2011) 1222 (DOI: 10.1109/ jqe.2011.2162398).

[11] K. Negishi and H. Uenohara: "Operational performance of an optical serial-to-parallel converter based on a Mach-Zehnder delay interferometer and a phase-shifted preamble for DPSK-formatted signals," IEICE Trans. Electron. E96-C (2013) 1012 (DOI: 10.1587/ transele.e96.c.1012).

[12] S. Nakamura, et al.: "Compact and low-loss $8 \times 8$ silicon photonic switch module for transponder aggregators in CDC ROADM application," Proc. Optical Fiber Commun. Conf. (OFC2015) (2015) M2B.6 (DOI: 10.1364/ofc.2015.m2b.6).

[13] S.-H. Kim, et al:: "Compact $2 \times 2$ polarization-diversity Si wire switch,”' Opt. Express 22 (2014) 29818 (DOI: 10.1364/oe.22.029818).

[14] H. Fukuda, et al.: "Ultrasmall polarization splitter based on silicon wire waveguides,” Opt. Express 14 (2006) 12401 (DOI: 10.1364/ oe.14.012401).

[15] L. Jia, et al.: "Compact optical polarization rotators based on and asymmetric silicon waveguide," IEEE Photon. Technol. Lett. 22 (2013) 2229 (DOI: 10.1109/lpt.2013.2284597).

[16] K. Morita and H. Uenohara: "Analytical and experimental investigation of a silicon photonic two-stage Mach-Zehnder delay interferometer-type polarization beam splitter," IEEE Photon. J. 10 (2018) (DOI: 10.1109/jphot.2018.2827043).

[17] Z. Su, et al.: "Four-port integrated polarization beam splitter," Opt. Lett. 39 (2014) 965 (DOI: 10.1364/ol.39.000965).

[18] Z. Lu, et al.: "Wideband silicon photonic polarization beamsplitter based-on point-symmetric cascaded broadband couplers," Opt. Express 23 (2015) 29413 (DOI: 10.1364/oe.23.029413).

[19] D. Chen, et al.: "Broadband, fabrication-tolerant polarization beam splitters based on a tapered directional coupler," IEEE Photon. Technol. Lett. 28 (2016) 2074 (DOI: 10.1109/lpt.2016.2583782).

[20] B. Ni and J. Xiao: "Ultracompact and broadband silicon-based polarization beam splitter using an asymmetric directional coupler," IEEE J. Quantum Electron. 53 (2017) (DOI: 10.1109/jqe.2017.2721539).

[21] H. Wu and D. Dai: "High-performance polarizing beam splitters based on cascaded bent directional couplers," IEEE Photon. Tehcnol. Lett. 29 (2017) 474 (DOI: 10.1109/lpt.2017.2657539).

[22] H. Wu, et al.: "Ultra-broadband high-performance polarizing beam splitter on silicon," Opt. Express 25 (2017) 6069 (DOI: 10.1364/ oe.25.006069).

[23] B. Bai, et al.: "Plasmonic-assisted polarization beam splitter based on bent directional coupling," IEEE Photon. Technol. Lett. 29 (2017) 599 (DOI: 10.1109/lpt.2017.2675448).

[24] J.R. Ong, et al.: "Broadband silicon polarization beam splitter with a high extinction ratio using a triple-bent-waveguide directional coupler,' Opt. Lett. 42 (2017) 4450 (DOI: 10.1364/ol.42.004450).

[25] A. Hosseini, et al.: "Ultracompact and fabrication-tolerant integrated polarization splitter," Opt. Lett. 36 (2011) 4047 (DOI: 10.1364/ ol.36.004047).

[26] M. Yin, et al.: "CMOS-compatible and fabrication-tolerant MMIbased polarization beam splitter," Opt. Commun. 335 (2015) 48 (DOI: 10.1016/j.optcom.2014.08.060).

[27] E.A. El-Fiky, et al.: "A high extinction ratio, broadband, and compact polarization beam splitter enabled by cascaded MMIs on silicon-oninsulator," Optical Fiber Communication Conference and Exposition 2016 (OFC2016) (2016) W2A.8 (DOI: 10.1364/ofc.2016.w2a.8).

[28] L. Xu, et al.: "Polarization beam splitter based on MMI coupler with SWG birefringence engineering on SOI," IEEE Photon. Technol. Lett. 30 (2018) 403 (DOI: 10.1109/lpt.2018.2794466).

[29] D. Dai, et al.: "Considerations for the design of asymmetrical MachZehnder interferometers used as polarization beam splitters on a submicrometer silicon-on-insulator platform," J. Lightw. Technol. 29 (2011) 1808 (DOI: 10.1109/jlt.2011.2142392).

[30] D. Dai, et al.: "Compact polarization beam splitter using an asymmetrical Mach-Zehnder interferometer based on silicon-on-insulator waveguides," IEEE Photon. Technol. Lett. 24 (2012) 673 (DOI: 10.1109/lpt.2012.2184530)

[31] J. Feng and R. Akimoto: "Silicon nitride polarizing beam splitter with potential application for intersubband-transition-based alloptical gate device," Jpn. J. Appl. Phys. 54 (2015) 04DG08 (DOI: 10.7567/jjap.54.04dg08).

[32] S. Gao, et al.: "Low-loss and broadband $2 \times 2$ polarization beam splitter based on silicon nitride platform," IEEE Photon. Technol. Lett. 28 (2016) 1936 (DOI: 10.1109/lpt.2016.2577605). 
[33] Y. Nasu, et al:: "Temperature insensitive and ultra wideband silicabased dual polarization optical hybrid for coherent receiver with highly symmetrical interferometer design," Proc. 37th European Conf. Opt. Commun. (ECOC2011) (2011) Tu.3.LeSalve.4 (DOI: 10.1364/ecoc.2011.tu.3.lesaleve.4). 